\section{Ecturs}

State University of Rio de
Janeiro, Pulmonology Service, Rio de Janeiro, Brazil

\section{Correspondence to} Dr Rogerio Rufino, State University of Rio de Janeiro, Pulmonology Service, Rua Mario Pederneiras, 10/121, Humaitá, Rio de Janeiro, RJ 22261-020, Brazil: rrufino.uerj@gmail.com

Received 15 December 2013 Revised 6 March 2014 Accepted 4 April 2014 Published Online First 24 April 2014

\title{
Fever, thoracic pain, skin lesions and Hampton hump
}

\author{
Germana Rocha Torres, Joana Acar, Ana Carolina Brito, Nelson Pimentel, \\ Nadja Polisseni, Cláudia Henrique da Costa, Rogério Rufino
}

\section{CASE PRESENTATION}

A 56-year-old white woman who had previously been healthy had been admitted to hospital 6 months previously for 2 months due to fever for 1 week associated with an onset of pain in her left hemithorax. She was initially treated for community-acquired pneumonia with a respiratory quinolone and continued to experience pain, which was worse during expiration. She lost $8 \mathrm{~kg}$ over the period of her admittance and developed plaque and papules. A chest X-ray showed opacity with fairly precise limits in the lower third of the left hemithorax similar to a Hampton hump, which consists of a shallow wedge-shaped opacity in the periphery of the lung with its base against the pleural surface, associated with diaphragmatic clamping (figure $1 \mathrm{~A}-\mathrm{C}$ ).

The laboratory findings included leukopenia with a discrete presence of monocytosis. D-dimer was within the normal value. Lower limb venous Doppler showed no venous thrombosis and the transthoracic echocardiogram was negative for vegetative lesions. Induced sputum was negative for tuberculosis and the tuberculin test was not reactive. Spirometry showed indices within normal limits. Serological markers for hepatitis B and C and anti-HIV 1 and 2 were negative. A skin biopsy was performed and suggested a framework associated with a lymphomatoid drug eruption.

A left pulmonary biopsy was performed and intense lymphocytic inflammatory infiltrate-forming follicles associated with alveolar epithelial hyperplasia and severe flaking occupying the alveoli were identified. Immunohistochemistry showed a heterogeneous population of lymphocytes that was not compatible with lymphoma. The patient was also tested for syphilis.

The patient was discharged and continued to be followed in the dermatology outpatient clinic where prednisone was started at a dose of $40 \mathrm{mg} /$ day for 5 days titrated to $10 \mathrm{mg} /$ day; no improvement was seen. The patient was referred to the pulmonology clinic, where a new review of skin and lung biopsies and new serology for infectious and rheumatic diseases was requested. A Venereal Disease Research Laboratory (VDRL) test was positive (1:256) and fluorescent treponemal antibody absorption test (FTA-Abs) reagent was present. A skin biopsy showed atrophy of the epidermis and infiltration of lymphocytes and plasma cells in the dermis (figure 2A). These findings were interpreted as a framework of lymphomatoid drug eruption.

A pulmonary biopsy of the left lower lobe identified intense inflammatory infiltration of lymphocytes and plasma cells forming follicles associated with alveolar epithelial hyperplasia and severe flaking occupying the alveoli. These inflammatory cells also appeared around vessels, forming a cuff. A nodule with characteristics of a typical gumma was present (figure 2B,C). These findings suggested pulmonary syphilis. Fontana-Tribondeau staining did not reveal any spirochaetes, and a diagnosis of lymphoma was excluded by immunohistochemical testing. Immunostaining for

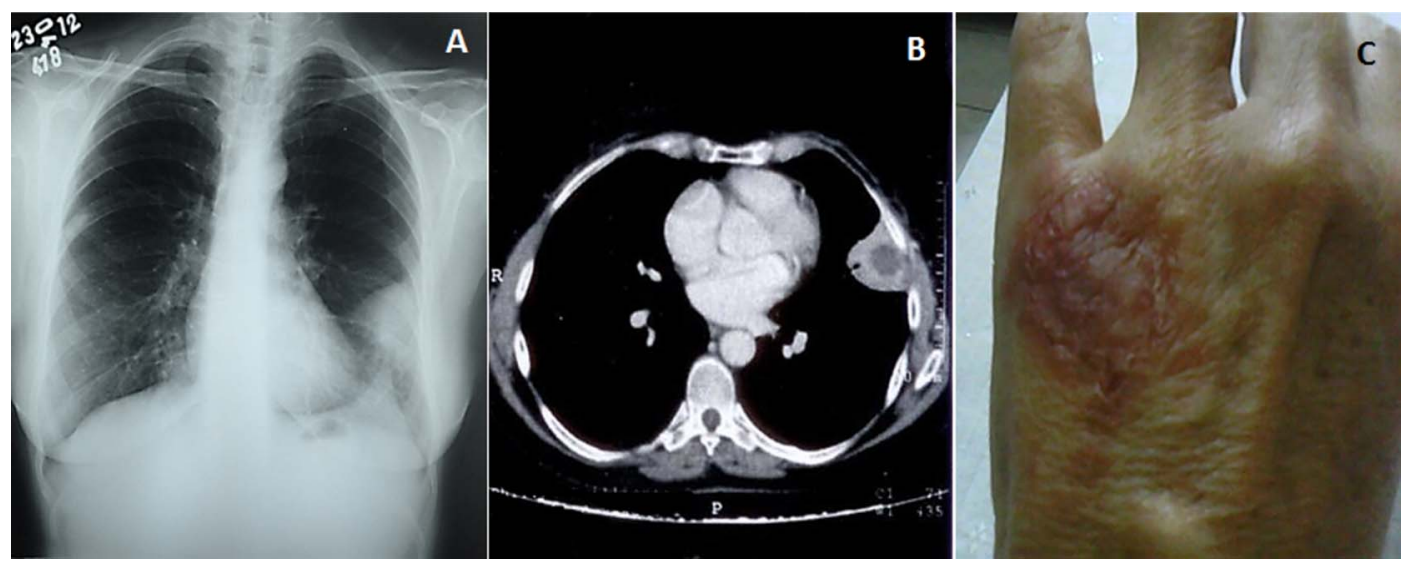

Figure 1 (A) Chest X-ray showing opacity in in the lower third of the left hemithorax and diaphragmatic clamping. (B) Chest CT scan showing opacity with soft tissue density. In the lingula, a heterogeneous broad-based deployment in the adjacent pleural surface measuring $3.6 \times 3.1 \mathrm{~cm}$ showed a hypodense area in the centre, which may correspond to the degenerate cystic/necrotic lobe. (C) Infiltrative non-pruritic erythematous plaque on the dorsum of the left hand. 


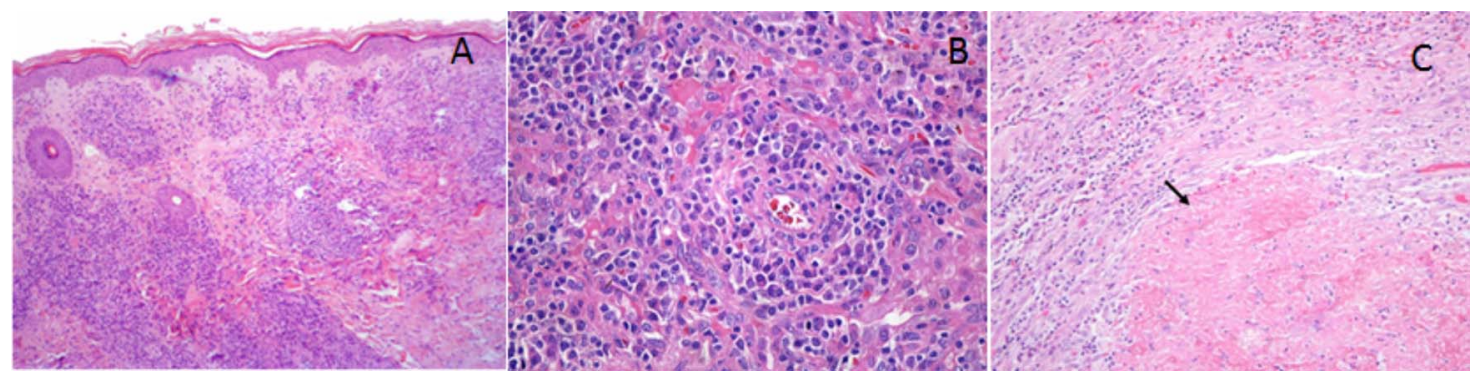

Figure 2 (A) Atrophic epidermis with vacuolar degeneration of the basal layer, extravasation of red blood cells and a superficial and mid-perivascular infiltrate in the dermis (H\&E 100x). (B) Blood vessel with a cuff of plasma cells and lymphocytes (H\&E 400x). (C) Pulmonary biopsy showing fibrosis and intense lymphoplasmocytic inflammatory infiltrate around a centre with a gumma (arrow) (H\&E 200x).

spirochaetes was not performed. The findings were consistent with syphilis (figure 2B,C).

\section{DISCUSSION}

Syphilis is a sexually transmitted infectious disease caused by a spirochaete bacterium Treponema pallidum. Its inoculation normally occurs without clinical manifestations. After 3 weeks the lesions (known as chancre) grow and merge with regional lymphadenopathy, characterising the primary stage of syphilis. Two to eight weeks later there is a new phase that is the secondary (or disseminated) stage of syphilis. Tertiary syphilis, characterised by chronic inflammation and progressive gumma, may develop years after the initial contact. ${ }^{12}$

The criteria for secondary syphilis are: (1) physical examination findings typical of secondary syphilis; (2) positive serological test for syphilis; (3) pulmonary abnormalities seen radiographically with or without signs and associated with pulmonary symptoms; (4) exclusion of other lung diseases; and (5) response to treatment with disappearance of the radiological findings. ${ }^{3}$ In this case, all stains to identify $T$ pallidum were negative and the skin lesion resembled gummatous syphilis. The patient was treated with penicillin and the skin lesions and radiographic images disappeared.

Syphilis is emerging in the world and should be included as a differential diagnosis of lung disease, especially in the presence of skin lesions.

Contributors GRT: acquisition of data, including the photo. JA: acquisition of data and obtained informed consent. ACB: acquisition of data and preparation of the histopathological case including the photos. NP: interpretation of data. NP: established the diagnosis and interpretation of data; CHdC: critical revision of article. RR: established the diagnosis, prepared the manuscript, reviewed and forwarded to Thorax and overall responsibility of content as guarantor.

Competing interests None.

\section{Patient consent Obtained.}

Provenance and peer review Not commissioned; externally peer reviewed.

\section{REFERENCES}

1 Scythes JB, Jones CM. Syphilis in the AIDS era: diagnostic dilemma and therapeutic challenge. Acta Microbiol Immunol Hung 2013;60:93-116.

2 Mattei PL, Beachkofsky TM, Gilson RT, et al. Syphilis: a reemerging infection. Am Fam Physician 2012;86:433-40.

3 Coleman DL, McPhee SJ, Ross TF, et al. Secondary syphilis with pulmonary involvement. West J Med 1983;138:875-8. 